\title{
SORET AND DUFOUR EFFECTS ON MHD FREE CONVECTIVE FLOW PAST A VERTICAL POROUS PLATE IN THE PRESENCE OF HEAT GENERATION
}

\author{
G.V.R. REDDY \\ Department of Mathematics, K.L. University \\ Guntur-522502, A.P, INDIA \\ E-mail: gvrr1976@kluniversity.in
}

\begin{abstract}
An MHD fluid flow is examined over a vertical plate in the presence of Dufour and Soret effects. The resulting momentum, energy and concentration equations are then made similar by introducing the usual similarity transformations. These similar equations are then solved numerically using the Runge-Kutta fourth order method with shooting technique. The effects of various parameters on the dimensionless velocity, temperature and concentration profiles as well as the local values of the skin-friction coefficient, the Nusselt number and Sherwood number are displayed graphically and in a tabular form. A comparison with previously published work is obtained and an excellent agreement is found.
\end{abstract}

Key words: radiation, heat and mass transfer, skin-friction, Nusselt number, Sherwood number.

\section{Introduction}

The effect of free convection on the accelerated flow of a viscous incompressible fluid past an infinite vertical plate with suction has many important technological applications in the astrophysical, geophysical and engineering problems. The heating of rooms and buildings by the use of radiators is a familiar example of heat transfer by free convection. Heat losses from the hot pipes, ovens, etc, heat and mass transfer play an important role in manufacturing industries for the design of fins, steel rolling, nuclear power plants, gas turbines and various propulsion devices for aircraft, combustion and furnace design, materials processing, energy utilization, temperature measurements. A comprehensive review of the studies of the convective heat transfer mechanism through porous media has been made by Nield and Bejan [1]. Hiremath and Patil [2] studied the effect of free convection currents on the oscillatory flow through a porous medium, which is bounded by a vertical plane surface of constant temperature. Fluctuating heat and mass transfer on a three-dimensional flow through a porous medium with variable permeability was discussed by Sharma et al. [3]. A comprehensive account of the available information in this field is provided in books by Pop and Ingham [4], Ingham and Pop [5], Vafai [6], Vadasz [7], etc.

Magnetohydrodynamics is currently undergoing a period of great development and differentiation of the subject matter. In the light of these applications, the steady MHD free convective flow past a heated vertical flat plate has been studied by many researchers such as Gupta [8], Lykoudis [9] and Nanda and Mohanty [10]. Chaudhary and Sharma [11] studied the effect of heat and mass transfer for a laminar mixed convection flow from a vertical surface with an induced magnetic field. El-Amin [12] studied the MHD free convection and mass transfer flow in a micropolar fluid over a stationary vertical plate with constant suction. The interest in these new problems follows from their importance in liquid metals, electrolytes and ionized gases. Many researchers have studied an MHD free convective heat and mass transfer flow in a porous medium. Some of them are Raptis and Kafoussias [13] who investigated heat and mass transfer effects on a steady MHD over a porous medium bounded by an infinite vertical porous plate with constant heat flux. Kim [14] found the effects of heat and mass transfer on an MHD micropolar flow over a vertical moving porous plate in a porous medium. 
At high operating temperatures, the radiation effect can be quite significant. Many processes in engineering areas occur at high temperatures and knowledge of the radiation heat transfer becomes very important for the design of the pertinent equipment. Nuclear power plants, gas turbines and various propulsion devices for aircrafts, missiles, satellites and space vehicles are examples of such engineering areas. The effect of radiation on an MHD flow and the heat transfer problem have become more important industrially. Singh and Agarwal [15] studied the heat transfer in a second grade fluid over an exponentially stretching sheet through a porous medium with thermal radiation and elastic deformation under the effect of a magnetic field. Makinde and Ogulu [16] studied the effect of thermal radiation on the heat and mass transfer flow of a variable viscosity fluid past a vertical porous plate permeated by a transverse magnetic field. Reddy and Reddy [17] analyzed the effects of an MHD oscillatory flow past a vertical porous plate embedded in a rotating porous medium. The study of heat generation in moving fluids is important as it changes the temperature distribution and the particle deposition rate, particularly in nuclear reactor cores, fire and combustion modeling, electronic chips and semiconductor wafers. Heat generation is also important in the context of exothermic or endothermic chemical reactions. Vajravelu and Hadjinicolaou [18] studied the heat transfer characteristics in the laminar boundary layer of a viscous fluid flow over a stretching sheet with viscous dissipation or frictional heating and internal heat generation. Hossain et al. [19] studied the problem of a natural convection flow along a vertical wavy surface with uniform surface temperature in the presence of heat generation/absorption. Kesavaiah et al. [20] reported on the effects of the chemical reaction and radiation absorption on an unsteady MHD convective heat and mass transfer flow past a semi-infinite vertical permeable moving plate embedded in a porous medium with heat source and suction. Reddy et al. [21] studied the unsteady MHD flow over a vertical moving porous plate with heat generation by considering double diffusive convection.

But in the above mentioned studies, Dufour and Soret terms have been neglected from the energy and concentration equations, respectively. It has been found that energy flux can be generated not only by the temperature gradient but also by the concentration gradient. The energy flux caused by the concentration gradient is called the Dufour effect and the same by the temperature gradient is called the Soret effect. These effects are very significant when the temperature and concentration gradients are very high. Anghel et al. [22] studied the Dufour and Soret effects on free convection boundary layer over a vertical surface embedded in a porous medium. Postelnicu [23] analyzed the influence of the magnetic field on heat and mass transfer from vertical surfaces in a porous media considering the Soret and Dufour effects. Alam et al. [24] investigated the Dufour and Soret effects on a steady MHD mixed convective and mass transfer flow past a semi-infinite vertical plate. Chamkha and Ben-Nakhi [25] analyzed the MHD mixed convection-radiation interaction along a permeable surface immersed in a porous medium in the presence of the Soret and Dufour effects. Many researchers have studied the Dufour and Soret effects on a free convective heat and mass transfer flow in a porous medium; some of them are Alam and Rahman [26], Sreedhar Sarma et al. [27], Mansour et al. [28], El-Aziz [29], Afify [30], Alam and Ahammad [31].

The aim of this paper is to discuss the Dufour and Soret effects on an MHD free convection flow past a vertical porous plate placed in a porous medium in the presence of chemical reaction, thermal radiation and heat source. The set of governing equations and boundary equation of the problem are transformed into a set of nonlinear ordinary differential equations with the help of similarity transformations are solved using the shooting method along with the fourth order Runge-Kutta integration scheme. The effects of different physical parameters on the velocity, temperature and concentration profiles as well as the local skin-friction coefficient, local Nusselt number and local Sherwood number are presented graphically and in a tabular form. To verify the obtained results, we have compared the present numerical results with previous work by Alam and Rahman [26]. The comparison results show a good agreement and we are confident that our present numerical results are accurate.

\section{Mathematical analysis}

A steady two-dimensional flow of an incompressible and electrical conducting viscous fluid, along an infinite vertical porous plate embedded in a porous medium is considered. The $x$ - axis is taken on the 
infinite plate, and parallel to the free-stream velocity which is vertical and the $y$-axis is taken normal to the plate. A magnetic field $B_{0}$ of uniform strength is applied transversely to the direction of the flow. Initially the plate and the fluid are at the same temperature $T_{\infty}$ in a stationary condition with concentration level $C_{\infty}$ at all points. The plate starts moving impulsively in its own plane with velocity $U_{0}$, its temperature is raised to $T_{w}$ and the concentration level at the plate is raised to $C_{w}$. The fluid is assumed to be slightly conducting, and hence the magnetic field is negligible in comparison with the applied magnetic field. It is further assumed that there is no applied voltage, so that the electric field is absent. The fluid is considered to be a gray, absorbing emitting radiation but non-scattering medium and the Roseland approximation is used to describe the radiative heat flux in the energy equation. It is also assumed that all the fluid properties are constant except that of the influence of the density variation with temperature and concentration in the body force term (Boussineq's approximation). Then, under the above assumptions, the governing boundary layer equations are

Continuity equation

$$
\frac{\partial u}{\partial x}+\frac{\partial v}{\partial y}=0
$$

Momentum equation

$$
u \frac{\partial u}{\partial x}+v \frac{\partial v}{\partial y}=v \frac{\partial^{2} u}{\partial y^{2}}+g \beta\left(T-T_{\infty}\right)+g \beta^{*}\left(C-C_{\infty}\right)-\frac{\sigma B_{0}^{2}}{\rho} u-\frac{v}{K} u-\frac{b}{K} u^{2}
$$

Energy equation

$$
u \frac{\partial T}{\partial x}+v \frac{\partial T}{\partial y}=\alpha \frac{\partial^{2} T}{\partial y^{2}}-\frac{1}{\rho c_{p}} \frac{\partial q_{r}}{\partial y}+\frac{D_{m}}{c_{s}} \frac{k_{T}}{c_{p}} \frac{\partial^{2} C}{\partial y^{2}}+\frac{Q_{0}}{\rho c_{p}}\left(T-T_{\infty}\right) .
$$

Concentration equation

$$
u \frac{\partial C}{\partial x}+v \frac{\partial C}{\partial y}=D_{m} \frac{\partial^{2} C}{\partial y^{2}}+\frac{D_{m} k_{T}}{T_{m}} \frac{\partial^{2} T}{\partial y^{2}}
$$

where $u, v$ are the Darcian velocities components in the $x$ and $y$ directions, respectively, $v$ is the kinematic viscosity, $g$ is the acceleration due to gravity, $\rho$ is the density, $\beta$ is the coefficient of volume expansion with temperature, $\beta^{*}$ is the volumetric coefficient of expansion with concentration, $b$ is the empirical constant, $T, T_{w}$ and $T_{\infty}$ are the temperature of the fluid inside the thermal boundary layer, the plate temperature and the fluid temperature in the free stream, respectively, $C, C_{w}$ and $C_{\infty}$ are the corresponding concentrations, $K$ is the Darcy permeability, $\sigma$ is the electric conductivity, $\alpha$ is the thermal diffusivity, $c_{p}$ is the specific heat at constant pressure, $k_{T}$ is the thermal diffusion ratio, $c_{s}$ is the concentration susceptibility, the term $Q_{0}\left(T-T_{\infty}\right)$ is assumed to be the amount of heat generated or absorbed per unit volume and $Q_{0}$ is a constant, which may take on either positive or negative values, $q_{r}$ is the radiative heat flux in the $y$-direction, $D_{m}$ is the coefficient of mass diffusivity. 
The boundary conditions for velocity, temperature and concentration fields are given by

$$
\begin{aligned}
& u=U_{0}, \quad v=v_{0}(x), \quad T=T_{w}, \quad C=C_{w} \quad \text { at } \quad y=0, \\
& u=0, \quad v=0, \quad T=T_{\infty}, \quad C=C_{\infty} \quad \text { as } \quad y \rightarrow \infty
\end{aligned}
$$

where $U_{0}$ is the uniform velocity and $v_{0}(x)$ is the velocity of suction at the plate.

We use the Rosseland approximation for radiation, radiative heat flux as given by Sparrow and Cess [32]

$$
q_{r}=-\frac{4 \sigma^{*}}{3 k^{*}} \frac{\partial T^{4}}{\partial y}
$$

where $\sigma^{*}$ and $k^{*}$ are the Stefan-Boltzman constant and the mean absorption coefficient, respectively. We assume that the temperature differences within the flow are such that the term $T^{4}$ may be expressed as a linear function of temperature. Hence, expending $T^{4}$ in a Taylor series about $T_{\infty}$ and neglecting higher order terms, we get

$$
T^{4} \equiv 4 T_{\infty}^{3} T-3 T_{\infty}^{4}
$$

Using Eqs (2.6) and (2.7) Eq.(2.3) becomes

$$
u \frac{\partial T}{\partial x}+v \frac{\partial T}{\partial y}=\alpha \frac{\partial^{2} T}{\partial y^{2}}+\frac{16 \sigma^{*} T_{\infty}^{3}}{3 k^{*}} \frac{1}{\rho c_{p}} \frac{\partial^{2} T}{\partial y^{2}}+\frac{D_{m}}{c_{s}} \frac{k_{T}}{c_{p}} \frac{\partial^{2} C}{\partial y^{2}}+\frac{Q_{0}}{\rho c_{p}}\left(T-T_{\infty}\right) .
$$

Equations (2.2), (2.4) and (2.8) are coupled, parabolic and nonlinear partial differential equations and hence an analytical solution is not possible. Therefore a numerical technique is employed to obtain the required solution. Numerical computations are greatly facilitated by non-dimensionalization of the equations. Proceeding with the analysis, we introduce the following similarity transformations and dimensionless variables which will convert the partial differential equations from two independent variables $(x, y)$ to a system of coupled, non-linear ordinary differential equations in a single variable ( $\eta$ ), i.e., a coordinate normal to the plate.

In order to write the governing equations and the boundary conditions in a dimensionless form, the following non-dimensional quantities are introduced.

$$
\eta=y \sqrt{\frac{U_{0}}{2 v x}}, \quad \psi=\sqrt{v x U_{0}} \quad f(\eta), \quad \theta(\eta)=\frac{T-T_{\infty}}{T_{w}-T_{\infty}}, \quad \phi(\eta)=\frac{C-C_{\infty}}{C_{w}-C_{\infty}}
$$

where $f(\eta)$ is the dimensionless stream function and $\psi$ is the dimensional stream function defined in the usual way

$$
u=\frac{\partial \psi}{\partial x} \quad \text { and } \quad v=-\frac{\partial \psi}{\partial y}
$$


Clearly, the continuity Eq.(2.1) is identically satisfied.

Then introducing relation (2.9) into Eq.(2.1) we obtain

$$
u=U_{0} f^{\prime}(\eta) \quad \text { and } \quad v=\sqrt{\frac{u U_{0}}{2 x}}\left(\eta f^{\prime}-f\right) .
$$

Further, introducing Eqs (2.9) and (2.10) into the momentum Eq.(2.2), energy Eq.(2.8) and concentration Eq.(2.4), we obtain the following local similarity equations

$$
\begin{aligned}
& f^{\prime \prime \prime}+f f^{\prime \prime}+\operatorname{Gr} \theta+\operatorname{Gc} \phi-M f^{\prime}-\frac{1}{\mathrm{DaRe}} f^{\prime}-\frac{F s}{\mathrm{Da}} f^{\prime 2}=0, \\
& \left(1+\frac{16}{3 R}\right) \theta^{\prime \prime}+\operatorname{Pr} f \theta^{\prime}+\operatorname{Pr} \operatorname{Du} \phi^{\prime \prime}+\operatorname{Pr} Q \theta=0, \\
& \phi^{\prime \prime}+\operatorname{Sc} f \phi^{\prime}+\operatorname{ScSr} \theta^{\prime \prime}=0
\end{aligned}
$$

where

$\mathrm{Gr}=\frac{g \beta\left(T_{w}-T_{\infty}\right) 2 x}{U_{0}^{2}}$ is the Grashof number, $\mathrm{Gc}=\frac{g \beta^{*}\left(C_{w}-C_{\infty}\right) 2 x^{2}}{v U_{0}}$ is the modified Grashof number, $M=\frac{\sigma B_{0}^{2} 2 x}{\rho U_{0}}$ is the magnetic field parameter, $\mathrm{Da}=\frac{K}{2 x^{2}}$ is the Darcy number, $\operatorname{Re}=\frac{U_{0} x}{v}$ is the Reynolds number, $\mathrm{Fs}=\frac{b}{x}$ is the Forchheimer number, $\operatorname{Pr}=\frac{v}{\alpha}$ is the Prandtl number, $R=\frac{k^{*} \alpha \rho c_{p}}{\sigma T_{\infty}^{3}}$ is the radiation parameter, $\mathrm{Du}=\frac{D_{m} K_{T}\left(C_{w}-C_{\infty}\right)}{c_{s} c_{p} \mathrm{v}\left(T_{w}-T_{\infty}\right)}$ is the Dufour number, $\mathrm{Sr}=\frac{D_{m} K_{T}\left(T_{w}-T_{\infty}\right)}{v T_{m}\left(C_{w}-C_{\infty}\right)}$ is the Soret number, $Q=\frac{Q_{0} \mathrm{U}}{\rho c_{p} U_{0}^{2}}$ is the heat generation parameter, $\mathrm{Sc}=\frac{\mathrm{v}}{D_{m}}$ is the Schmidt number.

The corresponding boundary conditions are

$$
\begin{aligned}
& f=f_{w}, \quad f^{\prime}=1, \quad \theta=1, \quad \phi=1 \quad \text { at } \quad \eta=0, \\
& f^{\prime}=0, \quad \theta=0, \quad \phi=0 \quad \text { as } \quad \eta \rightarrow \infty
\end{aligned}
$$

where $f_{w}=-v_{0} \sqrt{\frac{2 x}{v U_{0}}}$ is the dimensionless suction velocity and primes denote partial differentiation with respect to the variable $\eta$.

The parameters of engineering interest for the present problem are the skin-friction coefficient, the Nusselt number and the Sherwood number, which are given respectively by the following expressions. Knowing the velocity field, the skin-friction at the plate can be obtained, which in a non-dimensional form is given by

$$
\frac{1}{2} \operatorname{Re}^{\frac{1}{2}} C_{f}=f^{\prime \prime}(0)
$$


Knowing the temperature field, the rate of heat transfer coefficient can be obtained, which in a nondimensional form, in terms of the Nusselt number, is given by

$$
\mathrm{Nu} \operatorname{Re}^{-\frac{1}{2}}=-\theta^{\prime}(0)
$$

Knowing the concentration field, the rate of mass transfer coefficient can be obtained, which in a non-dimensional form, in terms of the Sherwood number, is given by

$$
\operatorname{Sh} \operatorname{Re}^{-\frac{1}{2}}=-\phi^{\prime}(0)
$$

where $\operatorname{Re}=\frac{U_{0} x}{v}$ is the Reynolds number.

\section{Mathematical solution}

The numerical solutions of the non-linear differential Eqs $(2.11)-(2.13)$ under the boundary conditions (2.14) have been performed by applying a shooting method along with the fourth order RungeKutta method. First of all, higher order non-linear differential Eqs (2.11) - (2.13) are converted into simultaneous linear differential equations of first order and they are further transformed into an initial value problem by applying the shooting technique. From this process of numerical computation, the skin-friction coefficient, the Nusselt number and Sherwood number which are respectively proportional to $f^{\prime \prime}(0),-\theta^{\prime}(0)$ and $-\phi^{\prime}(0)$ are also sorted out and their numerical values are presented in a tabular form.

\section{Results and discussion}

From the numerical computations, the dimensionless velocity, temperature and concentration profiles as well as the skin-friction coefficient, Nusselt number and Sherwood number are found for different values of the various physical parameters occurring in the problem. The value of the Prandtl number Pr is taken to be 0.71 which corresponds to air and the value of the Schmidt number Sc is chosen 0.22, which represents hydrogen at $25^{\circ} \mathrm{C}$ and $1 \mathrm{~atm}$. Due to the free convection problem positive large values of $\mathrm{Gr}=12$ and $\mathrm{Gc}=6$ are chosen. The value of Re is kept 100 and $F_{s}$ equal to 1.0. The values of the Dufour number and Soret number are chosen in such a way that their product is constant provided that the mean temperature $T_{m}$ is constant as well. However, the values of the Darcy number $\mathrm{Da}=1.0$, magnetic field parameter $M=1.0$, suction parameter $f_{w}=0.5$, radiation parameter $R=1.0$, heat generation parameter $Q=1.0$ are chosen arbitrarily. The numerical results for velocity, temperature and concentration profiles are displayed in Figs 2 to 13.

The effect of the Grashof number Gr on the velocity field is presented in Fig.1. The Grashof number Gr signifies the relative effect of the thermal buoyancy force to the viscous hydrodynamic force in the boundary layer. As the Grashof number Gr increases the velocity of the fluid increases. Figure 2 presents velocity profiles in the boundary layer for various values of the modified Grashof number Gc. The modified Grashof number Gc defines the ratio of the species buoyancy force to the viscous hydrodynamic force. As the modified Grashof number Gc increases the fluid velocity increases. The effect of the Darcy number Da on the temperature field is shown in Fig.3. From this figure we observe that velocity increases with the increase of the Darcy number Da. For a large Darcy number the porosity of the medium increases, hence the fluid flows quickly. The effect of the Reynolds number Re on the velocity fields is shown in Fig.4. It is observed that the negligible effect of the Reynolds number on velocity profiles. 


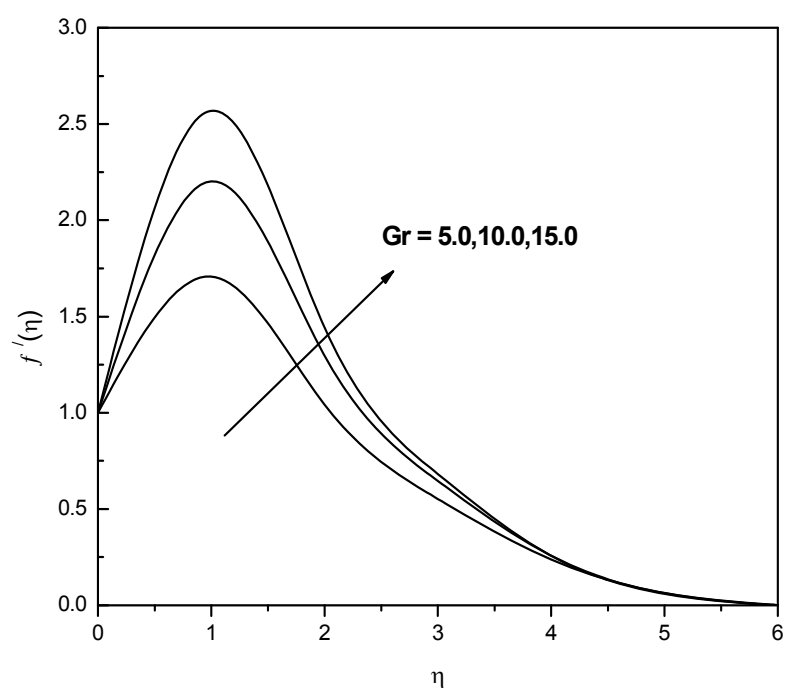

Fig.1. Velocity profiles for different values of Gr.

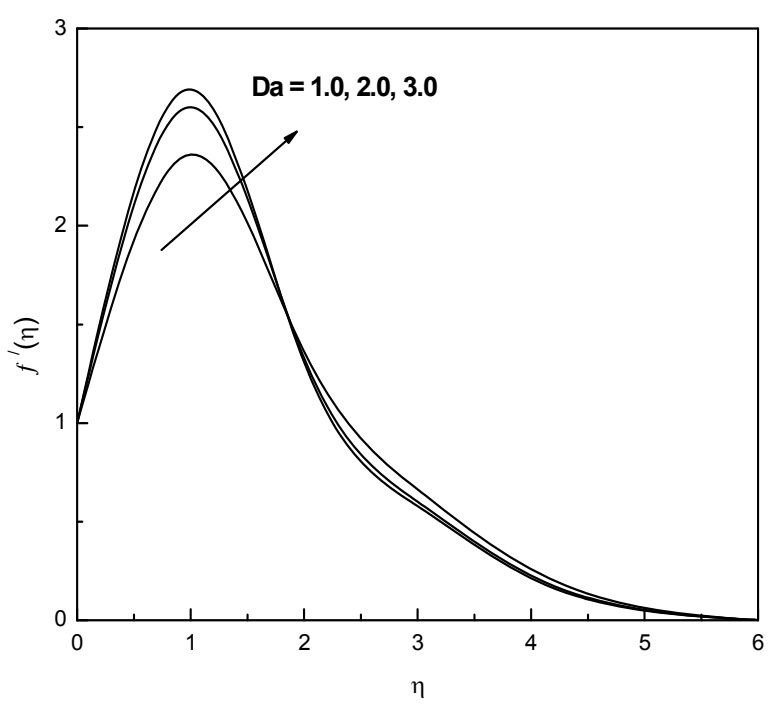

Fig.3. Velocity profiles for different values of Da.

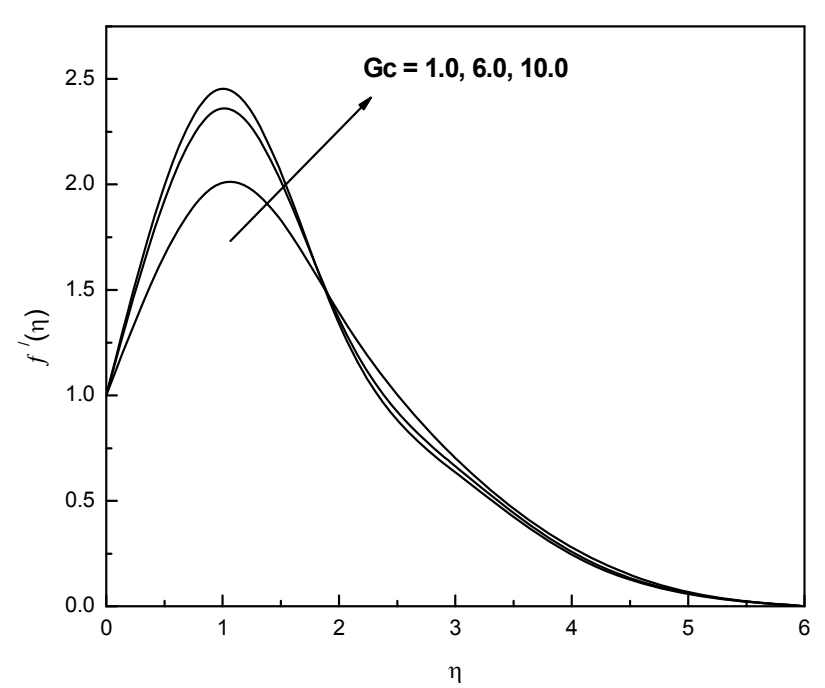

Fig.2. Velocity profiles for different values of Gc.

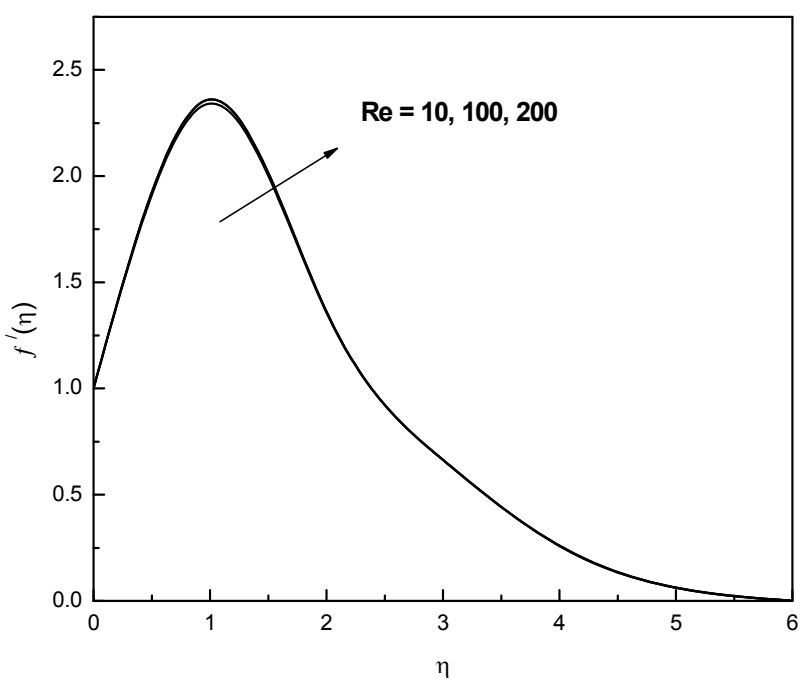

Fig.4. Velocity profiles for different values of Re.

Figures $5 \mathrm{a}-5 \mathrm{c}$ depict the effect of the Forchheimer number Fs on the velocity, temperature and concentration profiles. It is observed from Fig.5a that the velocity of the fluid decreases with the increase of the Forchheimer number Fs. Since the Forchheimer number Fs represents the inertial drag, thus an increase in the Forchheimer number Fs increases the resistance to the flow and so a decrease in the fluid velocity ensues. The temperature of the fluid increases with an increase of the Forchheimer number Fs, since the fluid is decelerated the energy is dissipated as heat and serves to increase the temperature. From Fig. $5 \mathrm{c}$, it is observed that the concentration of the fluid increases with an increase of the Forchheimer number Fs.

Figures $6 \mathrm{a}, 6 \mathrm{~b}$ and $6 \mathrm{c}$ display the velocity, temperature and concentration profiles for different values of the magnetic field parameter $M$ when the other parameters are fixed. An application of a magnetic field within the boundary layer has produced resistive-type force which is known as the Lorentz force. This force acts to retard the fluid motion along the surface and simultaneously increases its temperature and concentration values. Therefore, one can see that the velocity boundary layer thickness decreases with the increase of the magnetic field parameter $M$ as shown in Fig.6a. However, the temperature and concentration increase with the increasing of the magnetic field parameter $M$ shown in Figs $6 \mathrm{~b}$ and $6 \mathrm{c}$. 


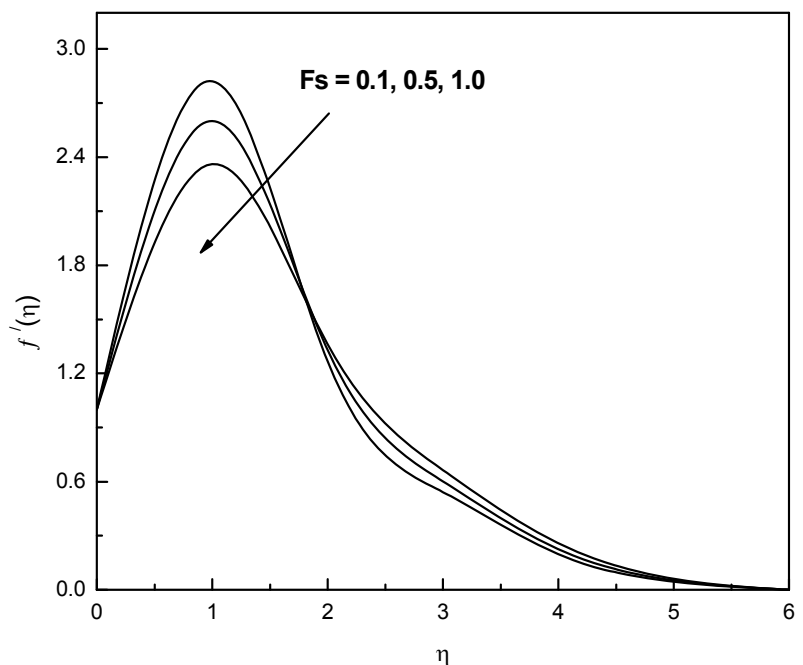

Fig.5a. Velocity profiles for different values of Fs.

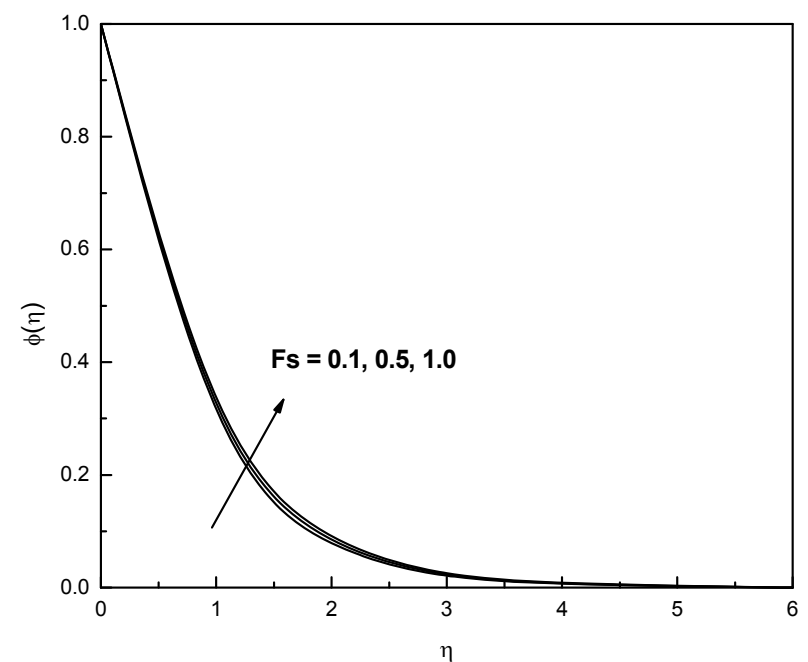

Fig.5c. Concentration profiles for different values of Fs.

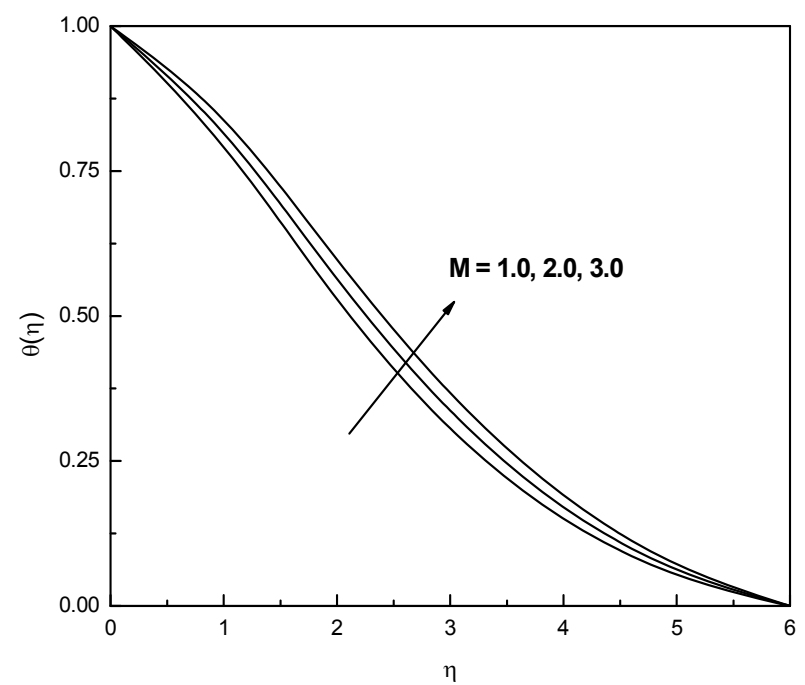

Fig.6b. Temperature profiles for different values of $M$.

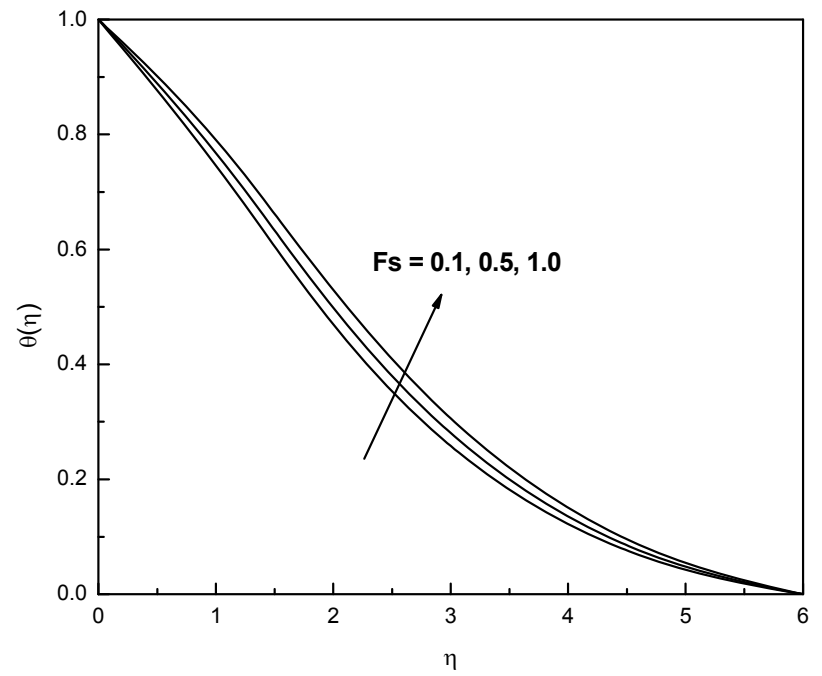

Fig.5b. Temperature profiles for different values of Fs.

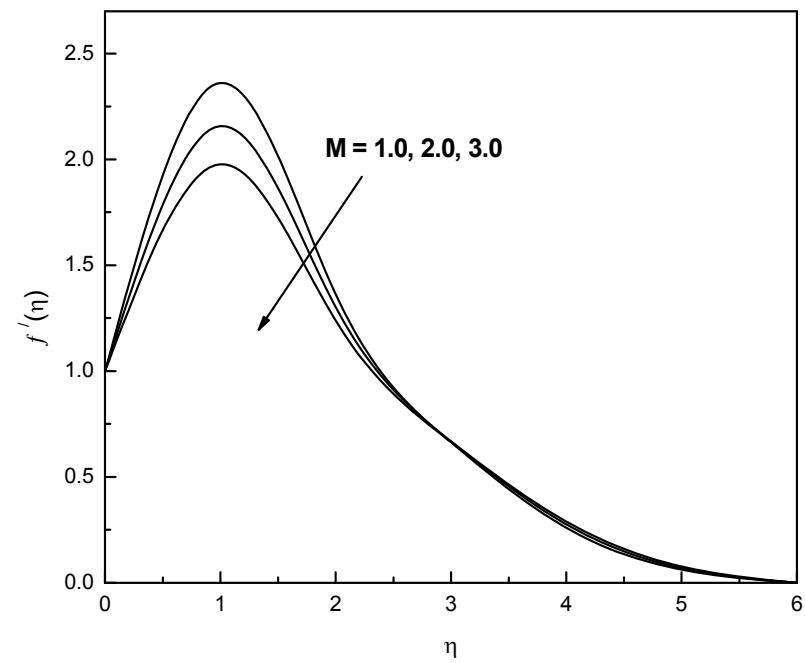

Fig.6a. Velocity profiles for different values of $M$.

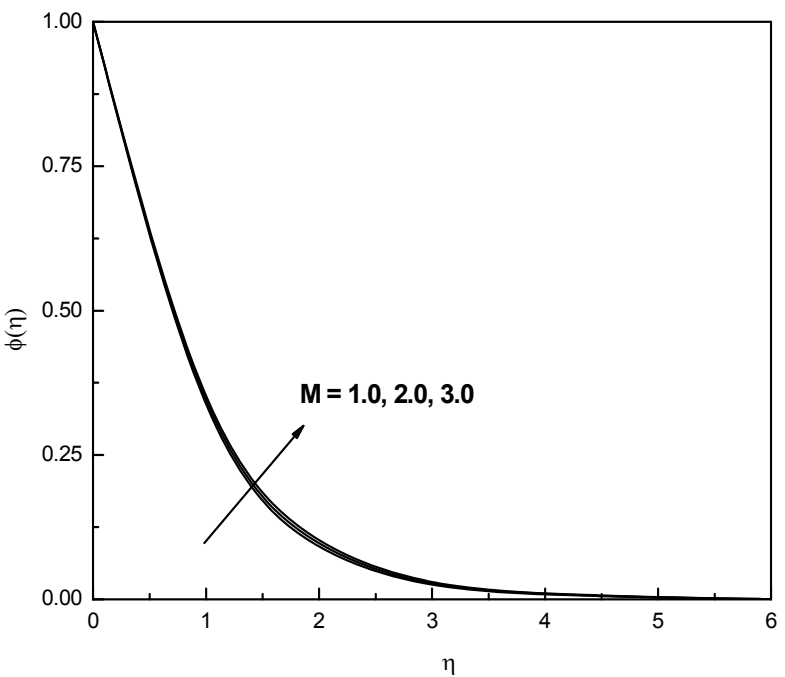

Fig.6c. Concentration profiles for different values of $M$. 
Figure 7a illustrates the velocity profiles for different values of the Prandtl number Pr. The numerical results show that the effect of increasing values of the Prandtl number results in a decreasing velocity. From Fig. $7 b$, it is observed that an increase in the Prandtl number results in a decrease of the thermal boundary layer thickness and in general lower average temperature within the boundary layer. The reason is that smaller values of Pr are equivalent to increasing the thermal conductivities, and therefore heat is able to diffuse away from the heated plate more rapidly than for higher values of Pr. Hence in the case of smaller Prandtl numbers the boundary layer is thicker and the rate of heat transfer is reduced.

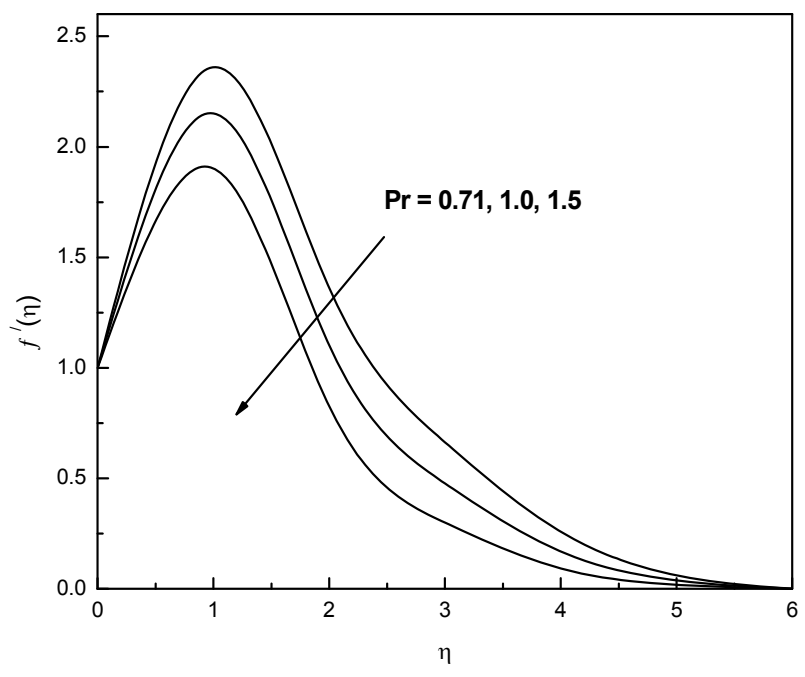

Fig.7a. Velocity profiles for different values of Pr.

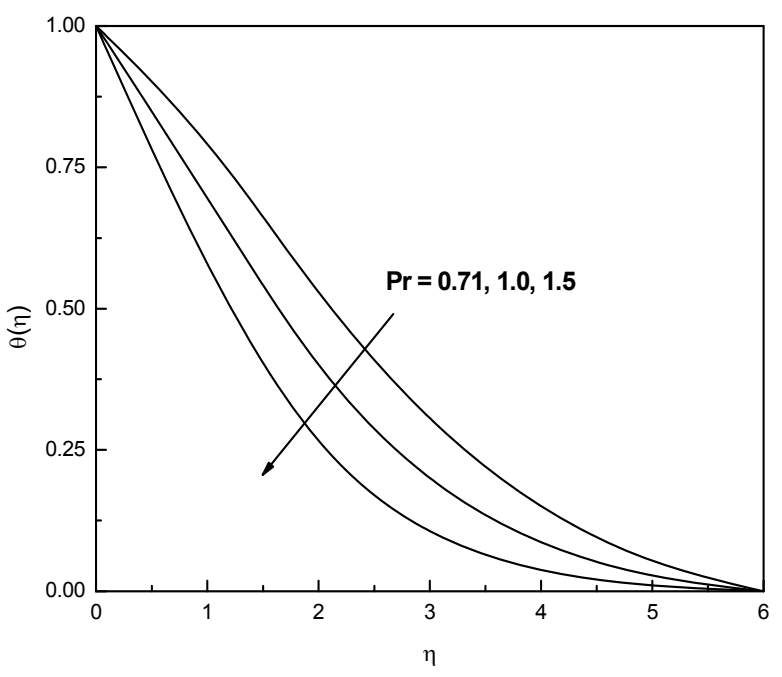

Fig.7b. Temperature profiles for different values of Pr.

The effect of the radiation parameter $R$ on the velocity and temperature profiles is shown in Figs $8 \mathrm{a}$ and $8 \mathrm{~b}$, respectively. Figure $8 \mathrm{a}$ shows that velocity profiles decrease with an increase in the radiation parameter $R$. From Fig.8b, it is seen that the temperature decreases as the radiation parameter $R$ increases. This result qualitatively agrees with expectations, since the effect of radiation is to decrease the rate of energy transport to the fluid, thereby decreasing the temperature of the fluid. Figures $9 \mathrm{a}$ and $9 \mathrm{~b}$ depict the velocity and temperature profiles for different values of the heat generation parameter $Q$. It is noticed that an increase in the heat generation parameter $Q$ results in an increase in velocity and temperature within the boundary layer.

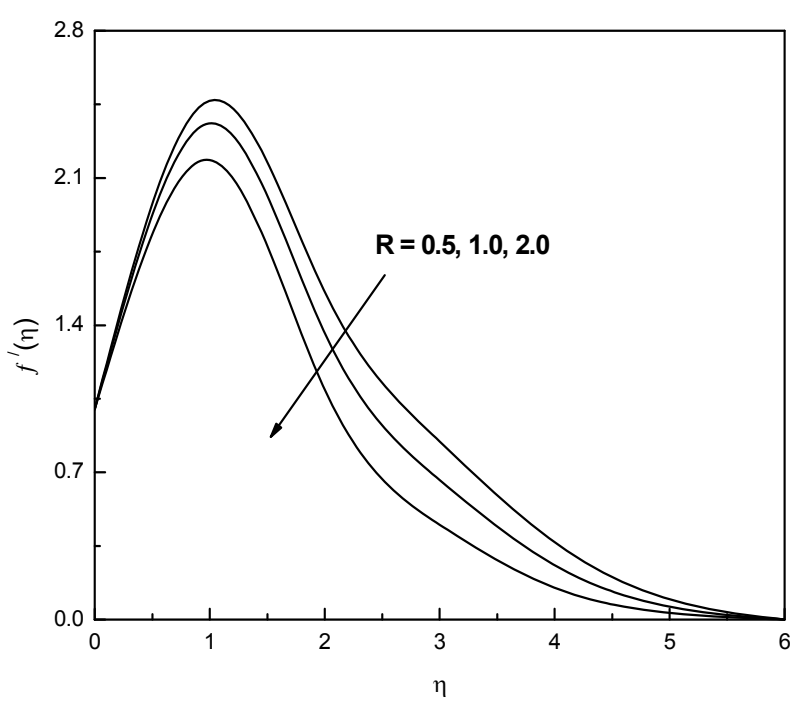

Fig.8a. Velocity profiles for different values of $R$.

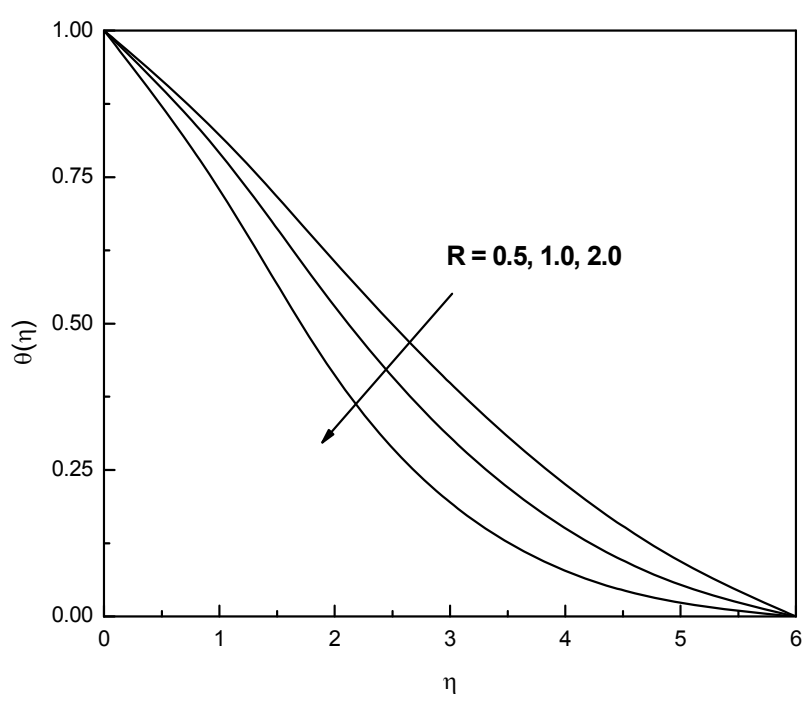

Fig.8b. Temperature profiles for different values of $R$. 


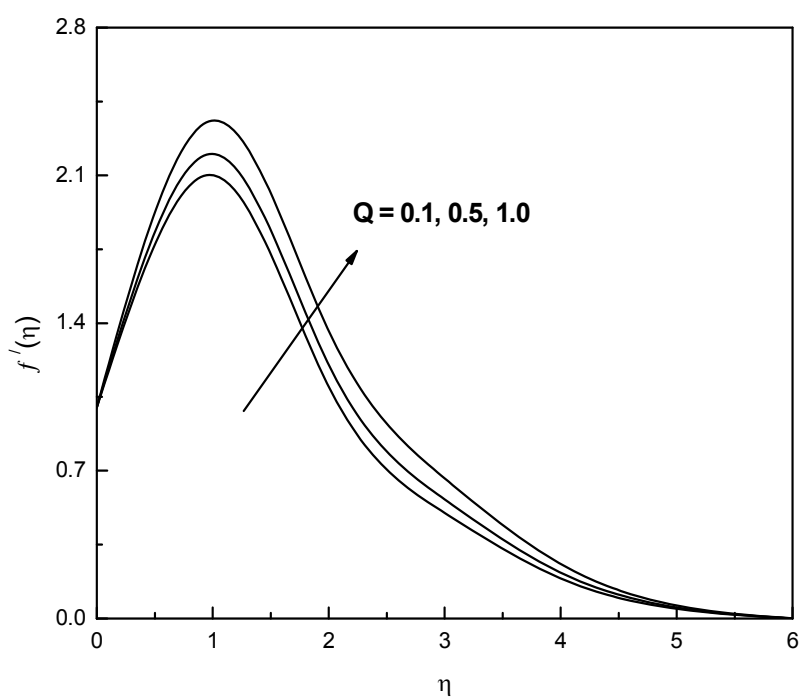

Fig.9a. Velocity profiles for different values of $Q$.

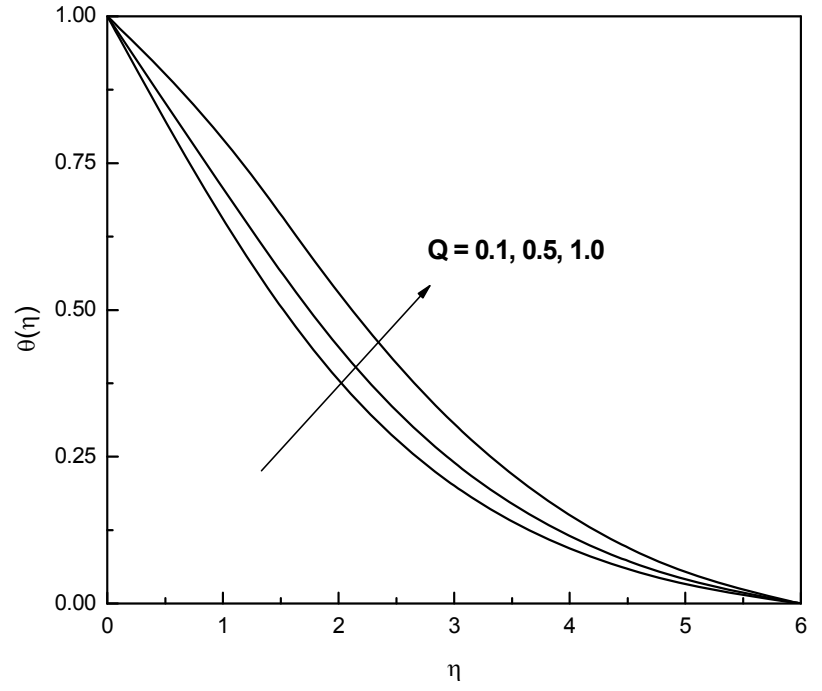

Fig.9b. Temperature profiles for different values of $Q$.

Figures 10a, 10b and 10c show the combined effects of the Dufour and Soret numbers on the fluid velocity, temperature and concentration respectively. The Dufour number Du and Soret number Sr represent the thermal- diffusion and diffusion-thermal effects in this problem. Figure 10a shows the influence of the Dufour and Soret number on the variations of the fluid velocity. For the case of increasing the Dufour number and decreasing the Soret number, it is seen that the velocity profiles decreases. Figure 10b illustrates the effects of the Dufour and Soret number on the variations of the fluid temperature. We observe that for an increasing Dufour number and decreasing Soret number, the temperature profiles increase. The Dufour term describes the effect of concentration gradients as noted in Eq.(2.12), plays a vital role in assisting the flow and is able to increase thermal energy in the boundary layer. This is the evident for the increasing values in the fluid temperature as the Dufour number Du increases and the Soret number Sr decreases. In Fig. 10c, the increasing Dufour number Du and simultaneously decreasing Soret number $\mathrm{Sr}$ have significant effects on the concentration profiles. The Soret term exemplifies the temperature gradient effects on the variation of concentration as noted in Eq.(2.13). It is observed that as the Dufour number increases and Soret number decreases, the concentration values are found to decrease.

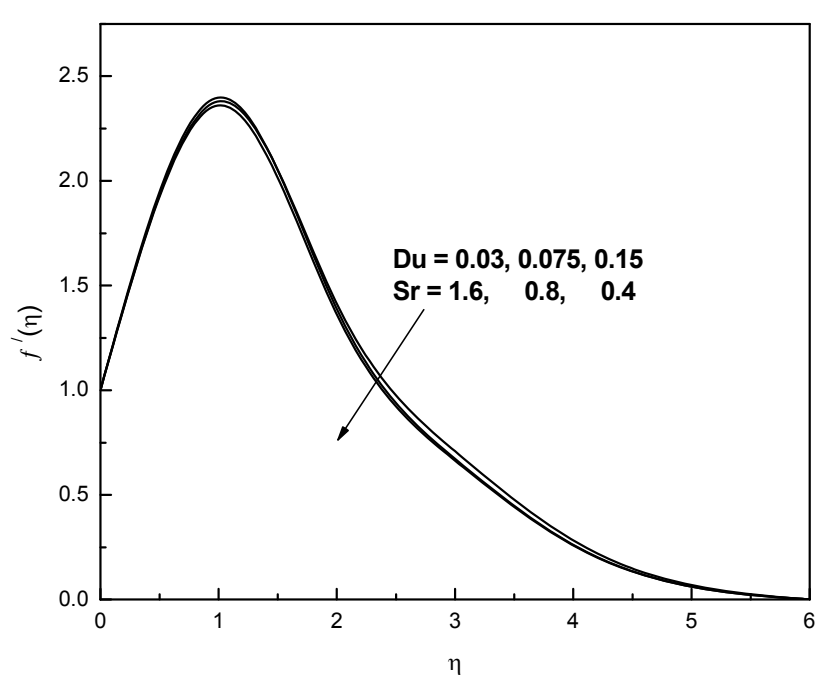

Fig.10a. Velocity profiles for different values of $\mathrm{Sr}$ and Du.

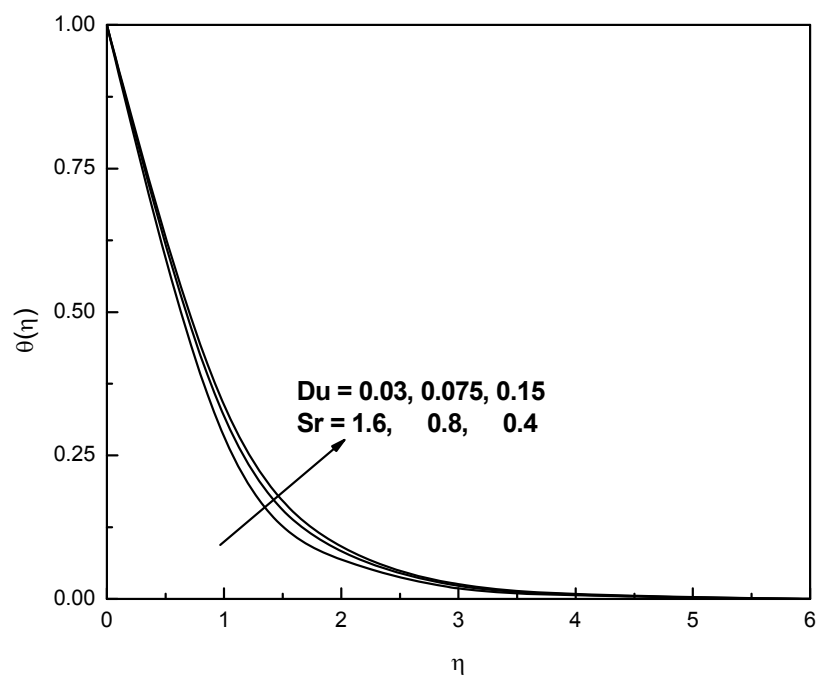

Fig.10b. Temperature profiles for different values of $\mathrm{Sr}$ and Du. 


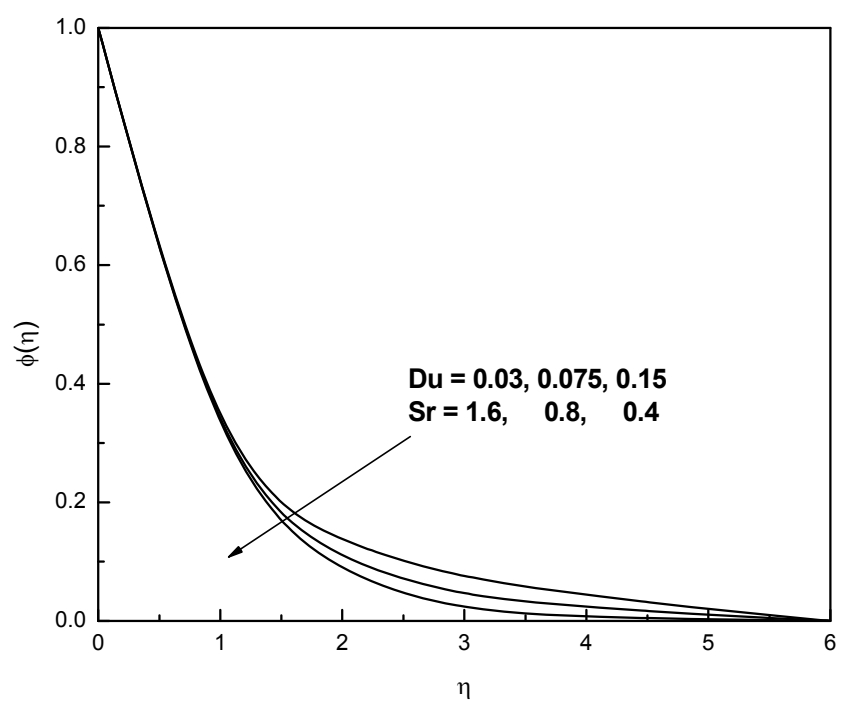

Fig.10c. Concentration profiles for different values of $\mathrm{Sr}$ and $\mathrm{Du}$.

The influence of the Schmidt number Sc on the velocity and concentration profiles is plotted in Figs $11 \mathrm{a}$ and $11 \mathrm{~b}$ respectively. As the Schmidt number Sc increases the concentration decreases. This causes the concentration buoyancy effects to decrease yielding a reduction in the fluid velocity. The reductions in the velocity and concentration profiles are accompanied by simultaneous reductions in the velocity and concentration boundary layers. These behaviors are clear from Figs 11a and 11b.

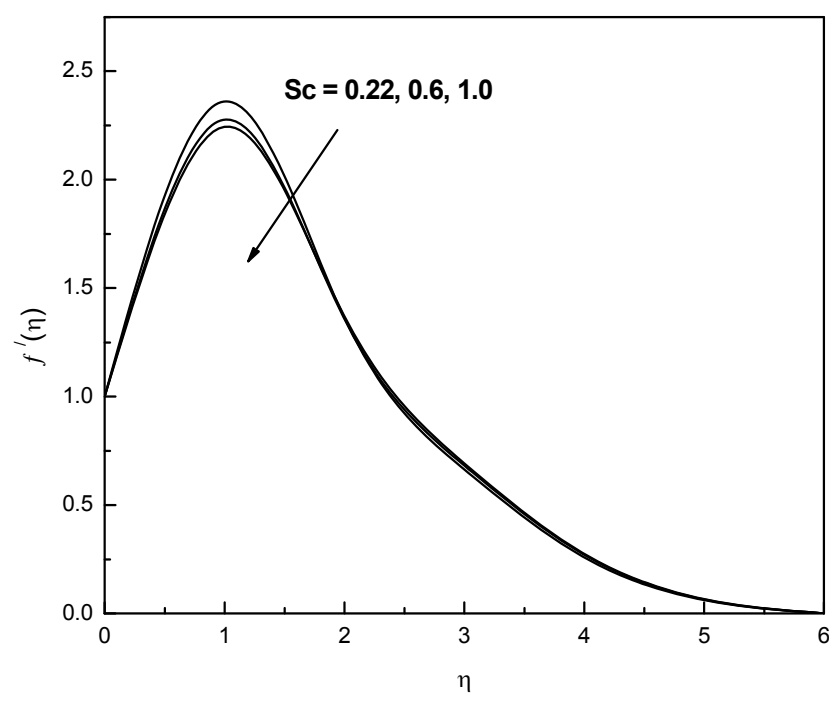

Fig.11a. Velocity profiles for different values of Sc.

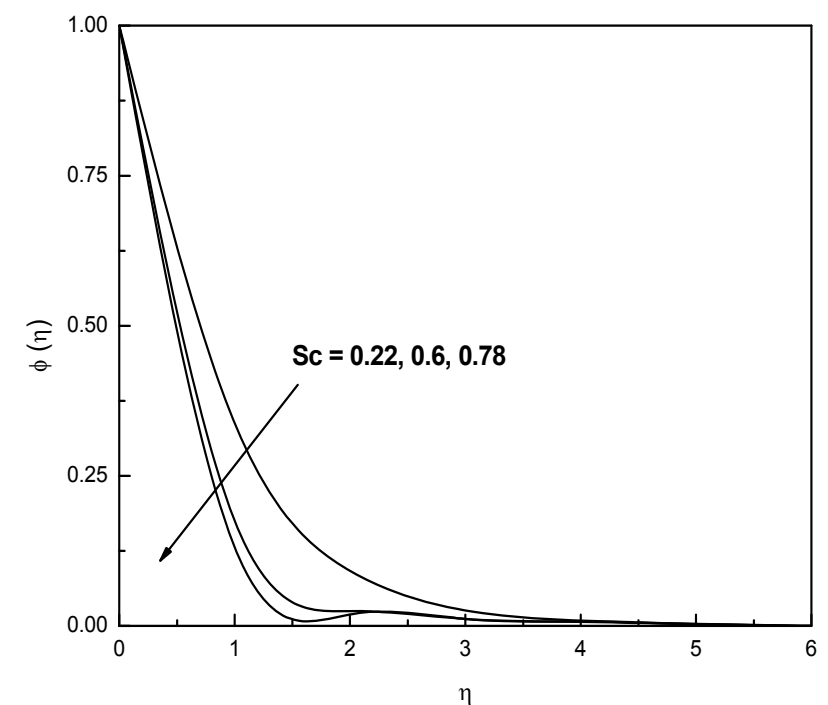

Fig.11b. Concentration profiles for different values of Sc.

The effects of the suction parameter $f_{w}$ on the velocity profiles are shown in Fig.12a. It is found from Fig.12a that the velocity profiles decrease monotonically with the increase of the suction parameter indicating the usual fact that suction stabilizes the boundary layer growth. The effects of the suction parameter on the temperature and concentration field are displayed in Fig.12b and Fig.12c respectively. From Fig. 12b, it is noticed that the temperature decreases with an increase of the suction parameter $f_{w}$. 
From Fig.12c, it is observed that the concentration decreases with an increase of the suction parameter $f_{w}$. The variations of the skin-friction coefficient, heat and mass transfer coefficient with the radiation parameter $R$ and the magnetic field parameter $M$ are shown in Figs 13a, 13b and 13c respectively. We observe that the effect of increasing $M$ is the decrease in the heat and mass transfer and skin friction coefficient. On the other hand, the magnitude of the heat and mass transfer increases while that of the skin friction coefficient decreases as the radiation parameter $R$ increases.
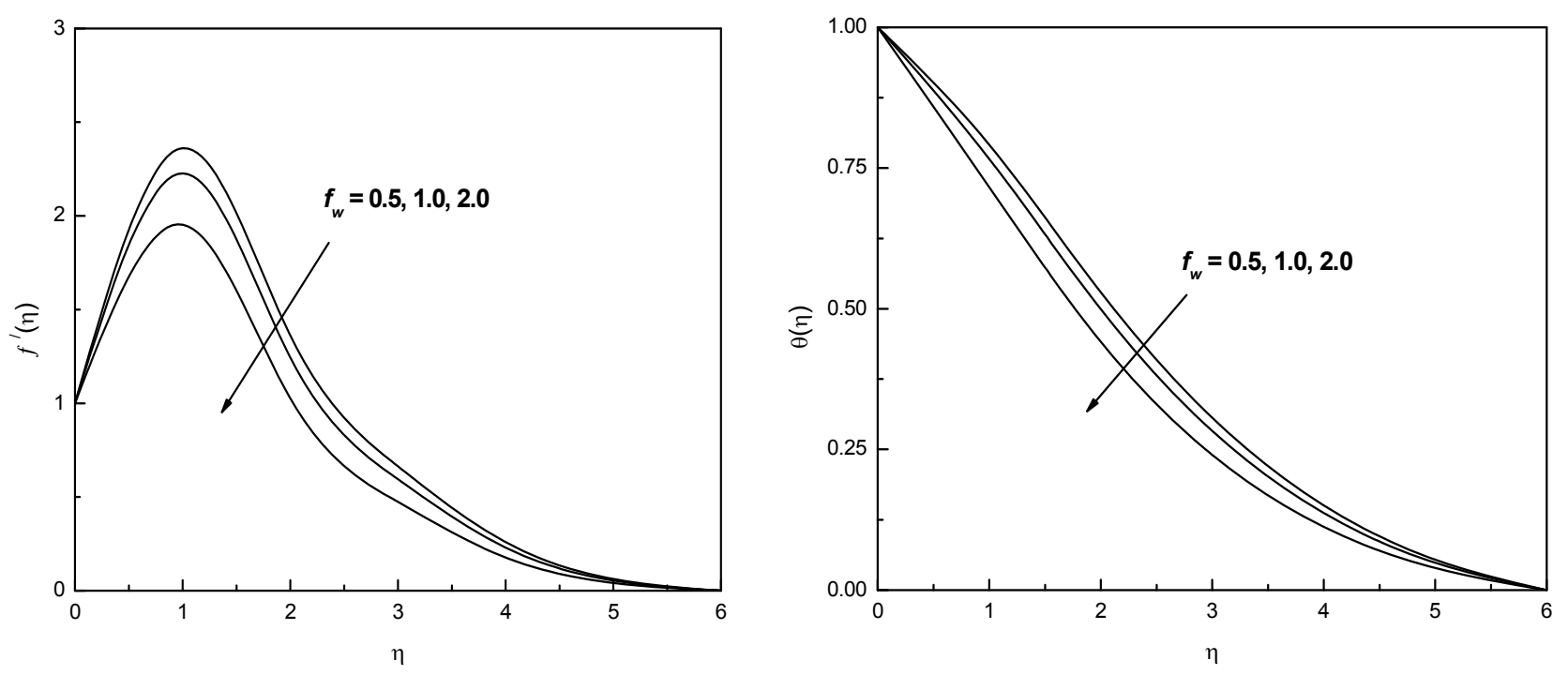

Fig.12a.Velocity profiles for different values of $f_{w}$. Fig.12b. Temperature profiles for different values of $f_{w}$.
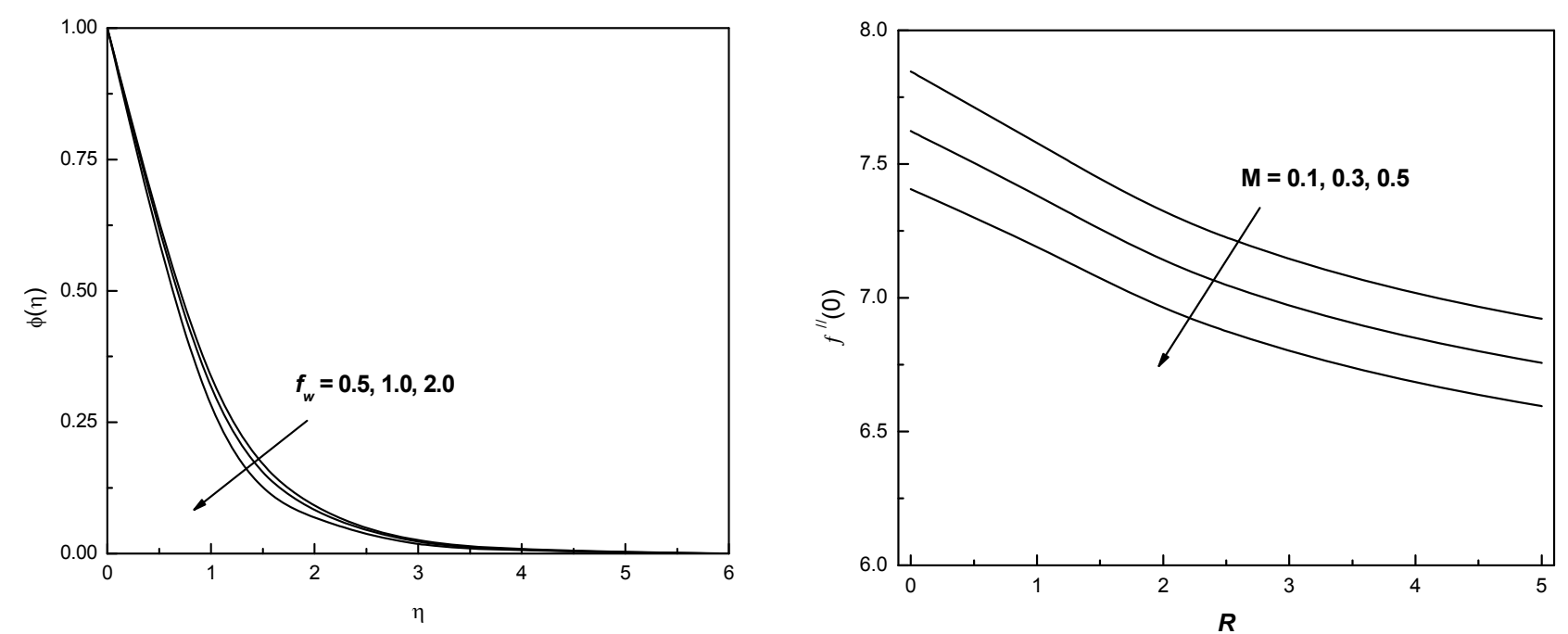

Fig.12c. Concentration profiles for different values of $f_{w}$.

Fig.13a. Variation of $f^{\prime \prime}(0)$ with $R$ and $M$. 

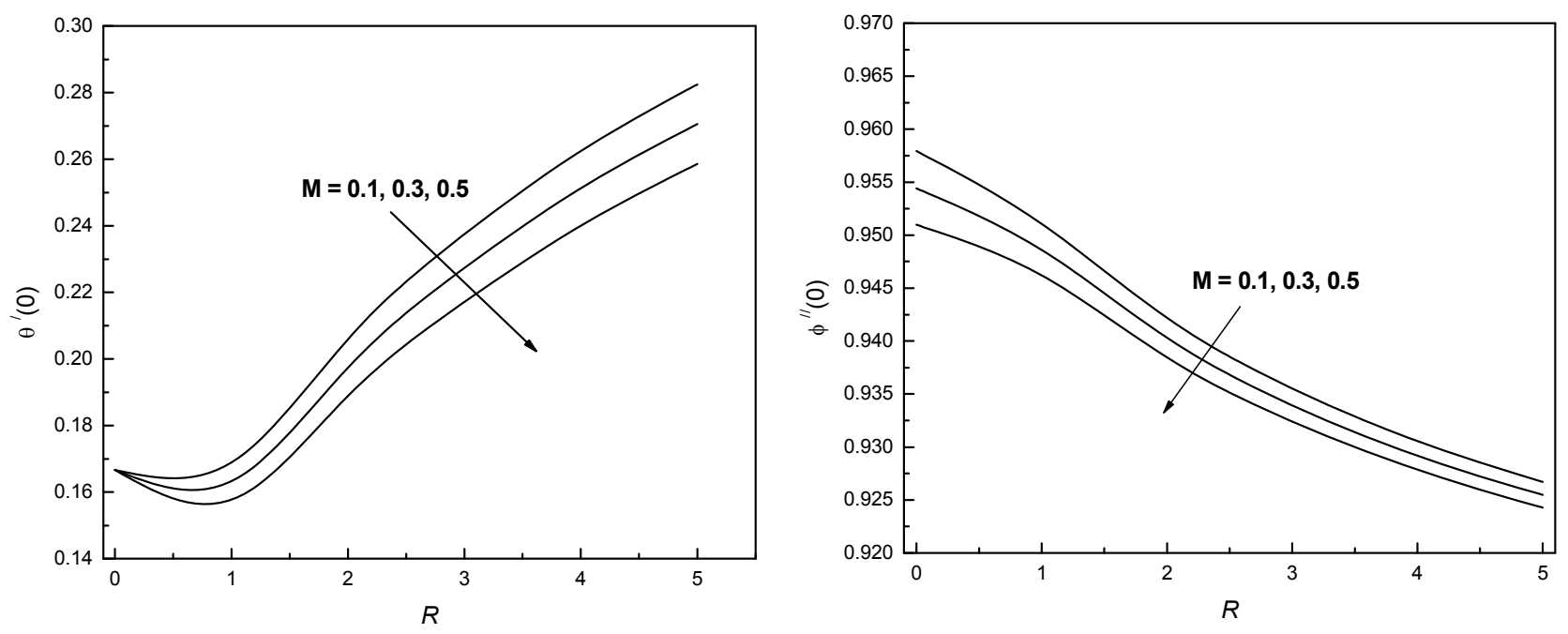

Fig.13b. Variation of the heat flux $-\theta^{\prime}(0)$ with $R$ and $M$. Fig.13c. Variation of the mass flux $-\phi^{\prime}(0)$ with $R$ and $M$.

Table 1 shows the comparison of $f^{\prime \prime}(0),-\theta^{\prime}(0)$ and $-\phi^{\prime}(0)$ with those reported by Alam and Rahman [26], which shows a good agreement and we are confident that our present numerical results are correct.

Tables 2, and 3 show the effects of the Grashof number Gr, the modified Grashof number Gc, the Darcy number Da, the magnetic parameter $M$, the suction parameter $f_{w}$, the Prandtl number Pr, the radiation parameter $R$, the heat generation parameter $Q$, and the Schmidt number Sc on the physical parameters of the skin-friction coefficient $f^{\prime \prime}(0)$, the Nusselt number $-\theta^{\prime}(0)$ and the Sherwood number $-\phi^{\prime}(0)$ respectively. It can be seen that all of $f^{\prime \prime}(0),-\theta^{\prime}(0)$ and $-\phi^{\prime}(0)$ increase as the Grashof number Gr, modified Grashof number Gc, Darcy number Da, and suction parameter $f_{w}$ increase. $f^{\prime}(0),-\theta^{\prime}(0)$ and $-\phi^{\prime}(0)$ decrease as the magnetic field parameter $M$ increases. $-\theta^{\prime}(0)$ increases as the Prandtl number Pr or radiation parameter $R$ increases, while it decreases with the heat generation parameter $Q .-\phi^{\prime}(0)$ increase as the Schmidt number $\mathrm{Sc}$ increases. Finally, the effects of the Soret number $\mathrm{Sr}$ and Dufour number Du on the skin-friction coefficient, Nusselt number and Sherwood number are shown in Tab.4. The behavior of these parameters is self-evident from Tab.4 and hence they will not be discussed any further due to brevity.

Table 1. Comparison values of $f^{\prime \prime}(0),-\theta^{\prime}(0)$ and $-\phi^{\prime}(0)$ with different values of the Soret and Dufour numbers.

\begin{tabular}{|c|c|c|c|c|c|c|c|}
\hline \multirow{2}{*}{$\mathrm{Sr}$} & \multirow{2}{*}{$\mathrm{Du}$} & \multicolumn{2}{|c|}{$f^{\prime \prime}(0)$} & \multicolumn{2}{c|}{$-\theta^{\prime}(0)$} & \multicolumn{2}{c|}{$-\phi^{\prime}(0)$} \\
\cline { 3 - 8 } & $\begin{array}{c}\text { Alam and } \\
\text { Rahman [26] }\end{array}$ & Present & $\begin{array}{c}\text { Alam and } \\
\text { Rahman [26] }\end{array}$ & Present & $\begin{array}{c}\text { Alam and } \\
\text { Rahman [26] }\end{array}$ & Present \\
\hline 2.0 & 0.03 & 3.4231141 & 3.42938 & 1.0283189 & 1.02699 & 0.1296854 & 0.12749 \\
\hline 1.0 & 0.06 & 3.3457474 & 3.35237 & 1.0155338 & 1.01386 & 0.2992750 & 0.297643 \\
\hline 0.5 & 0.12 & 3.3162482 & 3.32295 & 1.0019868 & 1.00022 & 0.3844602 & 0.382995 \\
\hline 0.4 & 0.15 & 3.3141130 & 3.32085 & 0.9965224 & 0.994735 & 0.4017999 & 0.400363 \\
\hline 0.2 & 0.30 & 3.3287043 & 3.33558 & 0.9718535 & 0.969957 & 0.4381199 & 0.436742 \\
\hline 0.1 & 0.60 & 3.3828661 & 3.38997 & 0.9248360 & 0.922756 & 0.4602605 & 0.458919 \\
\hline
\end{tabular}


Table 2. Numerical values of the skin-friction coefficient $(C f)$, Nusselt number $(\mathrm{Nu})$ and Sherwood number (Sh) for, $\operatorname{Pr}=0.71, \mathrm{Fs}=1.0, \mathrm{Re}=100, R=1.0, \mathrm{Du}=0.12, \mathrm{Sr}=0.5, \mathrm{Sc}=0.22, Q=1.0$.

\begin{tabular}{|c|c|c|c|c|c|c|c|}
\hline $\mathrm{Gr}$ & $\mathrm{Gc}$ & $\mathrm{Da}$ & $M$ & $f_{w}$ & $C f$ & $\mathrm{Nu}$ & $\mathrm{Sh}$ \\
\hline 12 & 6.0 & 1.0 & 1.0 & 0.5 & 6.34454 & 0.277773 & 0.921803 \\
\hline 5 & 6.0 & 1.0 & 1.0 & 0.5 & 3.42049 & 0.287881 & 0.879048 \\
\hline 10 & 6.0 & 1.0 & 1.0 & 0.5 & 5.54205 & 0.297256 & 0.910304 \\
\hline 12 & 2.0 & 1.0 & 1.0 & 0.5 & 5.05881 & 0.288464 & 0.907465 \\
\hline 12 & 4.0 & 1.0 & 1.0 & 0.5 & 5.70716 & 0.297754 & 0.914683 \\
\hline 12 & 6.0 & 2.0 & 1.0 & 0.5 & 7.43037 & 0.246511 & 0.941017 \\
\hline 12 & 6.0 & 3.0 & 1.0 & 0.5 & 7.90166 & 0.289481 & 0.949453 \\
\hline 12 & 6.0 & 1.0 & 2.0 & 0.5 & 5.50345 & 0.295472 & 0.907287 \\
\hline 12 & 6.0 & 1.0 & 3.0 & 0.5 & 4.76842 & 0.229296 & 0.894938 \\
\hline 12 & 6.0 & 1.0 & 1.0 & 1.0 & 6.65476 & 0.307874 & 0.981984 \\
\hline 12 & 6.0 & 1.0 & 1.0 & 2.0 & 7.12034 & 0.375616 & 1.10796 \\
\hline
\end{tabular}

Table 3. Numerical values of the skin-friction coefficient $(C f)$, Nusselt number $(\mathrm{Nu})$ and Sherwood number $(\mathrm{Sh})$ for $\mathrm{Gr}=12.0, \mathrm{Gc}=6.0, \mathrm{Da}=1.0, M=1.0, f_{w}=0.5, \mathrm{Du}=0.12, \mathrm{Sr}=0.5$.

\begin{tabular}{|c|c|c|c|c|c|c|}
\hline $\operatorname{Pr}$ & $R$ & $Q$ & $\mathrm{Sc}$ & $C f$ & $\mathrm{Nu}$ & $\mathrm{Sh}$ \\
\hline 0.71 & 1.0 & 1.0 & 0.22 & 6.34454 & 0.277773 & 0.921803 \\
\hline 1.0 & 1.0 & 1.0 & 0.22 & 6.1504 & 0.355013 & 0.910383 \\
\hline 1.5 & 1.0 & 1.0 & 0.22 & 5.8776 & 0.470762 & 0.893565 \\
\hline 0.71 & 2.0 & 1.0 & 0.22 & 6.06827 & 0.34458 & 0.909912 \\
\hline 0.71 & 3.0 & 1.0 & 0.22 & 5.90103 & 0.389547 & 0.902282 \\
\hline 0.71 & 1.0 & 0.1 & 0.22 & 6.27499 & 0.366377 & 0.911571 \\
\hline 0.71 & 1.0 & 0.5 & 0.22 & 6.3056 & 0.327556 & 0.916059 \\
\hline 0.71 & 1.0 & 1.0 & 0.6 & 6.09895 & 0.277768 & 1.26436 \\
\hline 0.71 & 1.0 & 1.0 & 0.78 & 6.01588 & 0.277751 & 1.41114 \\
\hline
\end{tabular}

Table 4. Numerical values of the skin-friction coefficient ( $C f)$, Nusselt number $(\mathrm{Nu})$ and Sherwood number (Sh) for, $\mathrm{Gr}=12.0, \mathrm{Gc}=6.0, \mathrm{Da}=1.0, M=1.0, f_{w}=0.5, \mathrm{Du}=0.12, \mathrm{Sr}=0.5, \mathrm{Pr}=$ $0.71, \mathrm{Fs}=1.0, \mathrm{Re}=100, R=1.0, \mathrm{Sc}=0.22, Q=1.0$.

\begin{tabular}{|c|c|c|c|c|}
\hline $\mathrm{Sr}$ & $\mathrm{Du}$ & $C f$ & $\mathrm{Nu}$ & $\mathrm{Sh}$ \\
\hline 0.5 & 0.12 & 6.34454 & 0.277773 & 0.921803 \\
\hline 1.0 & 0.12 & 6.35474 & 0.278037 & 0.920257 \\
\hline 2.0 & 0.12 & 6.37527 & 0.278545 & 0.917239 \\
\hline 0.5 & 0.03 & 6.3325 & 0.284933 & 0.920915 \\
\hline 0.5 & 0.06 & 6.33651 & 0.282548 & 0.921211 \\
\hline
\end{tabular}

\section{Conclusions}

In this paper, a mathematical model has been presented for studying the influence of radiation and heat generation on an MHD free convective flow past a vertical porous plate in a porous medium under the influence of Dufour and Soret effects. Using the similarity transformation a set of ordinary differential 
equations was derived for the conservation of mass, momentum and species diffusion in the boundary layer. These nonlinear, coupled differential equations were solved under valid boundary conditions using the fourth order Runge-Kutta method with the shooting technique. The conclusions of the study are as follows:

- The velocity increases with an increase of the Grashof number and modified Grashof number.

- The velocity decreases with an increase in the magnetic field parameter and permeability parameter.

- The temperature and velocity of the fluid increase with an increase of the radiation parameter.

- The temperature and velocity of the fluid increase with an increase of the heat source parameter.

- As the radiation parameter, the skin-friction coefficient and Nusselt number decrease.

- As the Dufour and Soret number increases, it is seen that the temperature profiles increase.

- As the Schmidt number Sc increases, the concentration decreases.

\section{Nomenclature}

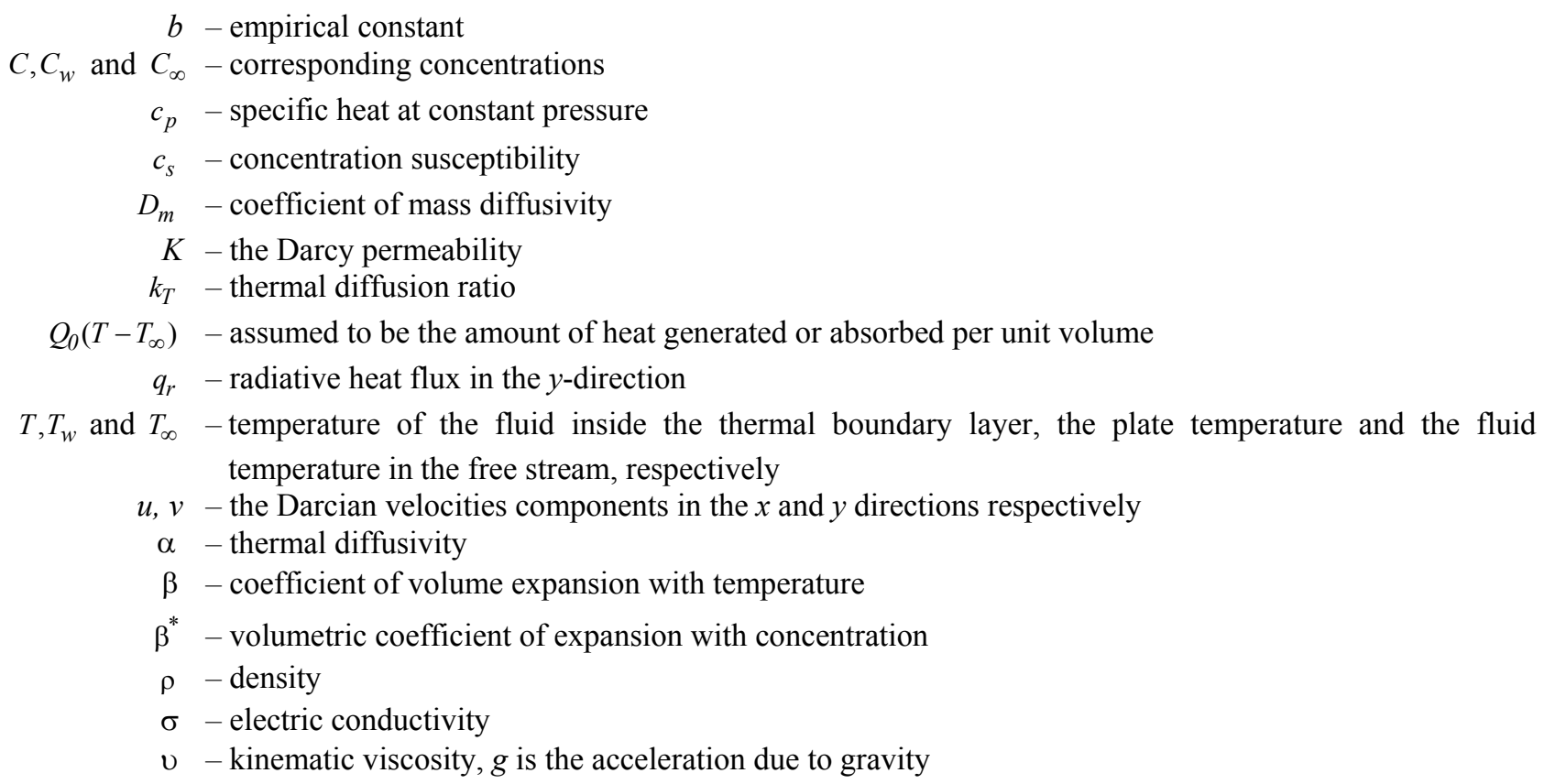

\section{References}

[1] Nield D.A. and Bejan A. (1998): Convection in Porous Media. - 2nd Edition, Berlin: Springer-Verlag.

[2] Hiremath P.S. and Patil P.M. (1993): Free convection effects on oscillatory flow of couple stress field through a porous medium. - Acta Mechanica, vol.98, No.1-4, pp.143-158.

[3] Sharma B.K., Chaudhary R.C. and Sharma P.K. (2007): Fluctuating mass transfer on three-dimensional flow through a porous medium with variable permeability. - Advancesin Theoretical and Applied Mathematics, vol.2, No.3, pp.257-267.

[4] Pop I. and Ingham D.B. (2001): Convective Heat Transfer: Mathematical and Computational Modeling of Viscous Fluids and Porous Media. - Oxford, UK: Pergamon.

[5] Ingham D.B. and Pop I. (2005): Transport Phenomena in Porous Media III. - Oxford: Elsevier.

[6] Vafai K. (2005): Handbook of Porous Media. - 2nd Edition, New York, NY, USA: Taylor and Francis.

[7] Vadasz P. (2008): Emerging Topics in Heat and Mass Transfer in Porous Media. - New York: Springer.

[8] Gupta A.S. (1961): Steady and transient free convection of an electrically conducting fluid from a vertical plate in the presence of magnetic field. - Appl. Sci. Res., vol.9, No.A, pp.319-333. 
[9] Lykoudis P.S (1962): Natural convection of an electrically conducting fluid in the presence of a magnetic field. Int. J. Heat Mass Transfer, vol.5, pp.23-34.

[10] Nanda R.S and Mohanty H.K (1970): Hydromagnetic free convection for high and low Prandtl numbers. - J. Phys. Soc. Japan, vol.29, pp.1608-1618.

[11] Chaudhary R.C. and Sharma B.K. (2006): Combined heat and mass transfer by laminar mixed convection flow from a vertical surface with induced magnetic field. - Journal of Applied Physics, vol.99, No.3, pp.34901-34100.

[12] El-Amin M.F. (2001): Magnetohydrodynamic free convection and mass transfer flow in micropolar fluid with constant suction. - Journal of Magnetism and Magnetic Materials, vol.234, No.3, pp.567-574.

[13] Raptis A. and Kafoussias N.G. (1982): Magnetohydrodynamic free convection flow and mass transfer through porous medium bounded by an infinite vertical porous plate with constant heat flux. - Can. J. Phys., vol.60, No.12, pp.1725-1729.

[14] Kim Y.J. (2004): Heat and mass transfer in MHD micropolar flow over a vertical moving porous plate in a porous medium. - Transport in Porous Media, vol.56, No.1, pp.17-37.

[15] Singh V. and Agarwal S. (2012): Heat transfer in a second grade fluid over an exponentially stretching sheet through porous medium with thermal radiation and elastic deformation under the effect of magnetic field - Int. J. of Appl. Math and Mech., vol.8, No.4, pp.41-63.

[16] Makinde O.D and Ogulu A. (2008): The effect of thermal radiation on the heat and mass transfer flow of a variable viscosity fluid past a vertical porous plate permeated by a transverse magnetic field. - Chemical Engineering Communications, vol.195, No.12, pp.1575-1584.

[17] Reddy G.V.R. and Reddy N.B. (2011): Effects of MHD oscillatory flow past a vertical porous plate embedded in a rotating porous medium. - International Journal of Research and Reviews in Applicable Mathematics and Computer Science, vol.1, No.4.

[18] Vajravelu K. and Hadjinicolaou A. (1993): Heat transfer in a viscous fluid over a stretching sheet with viscous dissipation and internal heat generation. - Int. Comm. Heat Mass Transfer, vol.20, pp.417-430.

[19] Hossain M.A., Molla M.M. and Yaa L.S. (2004). Natural convection flow along a vertical wary surface temperature in the presence of heat generations /absorption. - Int. J. Thermal Science, vol.43, pp.157-163.

[20] Kesavaiah D.Ch., Satyanarayana P.V. and Venkataramana S. (2011): Effects of the chemical reaction and radiation absorption on unsteady MHD convective heat and mass transfer flow past a semi-infinite vertical permeable moving plate embedded in porous medium with heat source and suction. - Int. J. of Appl. Math. and Mech., vol.7, No.1, pp52-69.

[21] Reddy G.V.R., Murthy Ch.V.R. and Reddy N.B. (2011): MHD flow over a vertical moving porous plate with heat generation by considering double diffusive convection. - International Journal of Applied Mathematics and Mechanics, vol.7, No.1, pp.1-17.

[22] Anghel M., Takhar H.S. and Pop I. (2000): Dufour and Soret effects on free convection boundary layer over a vertical surface embedded in a porous medium. - Stud. Univ. Babes-Bolyai, Math., vol.45, pp.11-21.

[23] Postelnicu I. (2004): Influence of a magnetic field on heat and mass transfer by natural convection from vertical surfaces in porous media considering Soret and Dufour effects. - Int. J. Heat Mass Transfer, vol.47, pp.14671472.

[24] Alam M.S., Rahman M.M., Maleque M.A. and Ferdows M. (2006): Dufour and Soret effects on steady MHD combined free-forced convective and mass transfer flow past a semi-infinite vertical plate. - Thammasat Int. J. Sci. Technol., vol.11, No.2, pp.1-12.

[25] Chamkha A.J. and Ben-Nakhi A. (2008): MHD mixed convection-radiation interaction along a permeable surface immersed in a porous medium in the presence of Soret and Dufour's effects. - Heat Mass Transfer, vol.44, pp.845-856.

[26] Alam M.S. and Rahman M.M. (2005): Dufour and Soret effects on MHD free convective heat and mass transfer flow past a vertical flat plate embedded in a porous medium. - J. Naval Architecture and Marine Engineering, vol.2, No.1, pp.55-65. 
[27] Sarma G.S., Prasad R.K. and Govardhan K. (2013): The combined effect of chemical reaction, thermal radiation on steady free convection and mass transfer flow in a porous medium considering Soret and Dufour effects. IOSR Journal of Mathematics, vol.8, No.2, pp.67-87.

[28] Mansour M.A., El-anssary N.F. and Aly A.M. (2008): Effects of chemical reaction and thermally stratification on MHD free convection heat and mass transfer over a vertical stretching surface embedded in porous media considering Soret and Dufour number. - Journal of Chemical Engineering, vol.145, No.2, pp.340-345.

[29] El-Aziz M.A. (2008): Thermal-diffusion and diffusion-thermo effects on combined heat and mass transfer by hydromagnetic three-dimensional free convection over a permeable stretching surface with radiation. - Phys. Lett. A, vol.372, pp.263-272.

[30] Afify A.A. (2009): Similarity solution in MHD: Effects of thermal-diffusion and diffusion-thermo on free convective heat and mass transfer over a stretching surface considering suction or injection. - Commun. Nonlinear Sci. Numer. Simul., vol.14, pp.2204-2214.

[31] Alam M.S. and Ahammad M.U. (2011): Effects of variable chemical reaction and variable electric conductivity on free convective heat and mass transfer flow along an inclined stretching sheet with variable heat of Dufour and Soret effects. - Nonlinear Analysis: Modelling and Control, vol.16, No.1, pp.1-16.

[32] Sparrow E.M. and Cess R.D. (1978): Radiation Heat Transfer. - Augmented Edition, Hemisphere Publishing Corp, Washington, DC.

Received: July 18, 2015

Revised: June 23, 2016 